\title{
New three-parameter correlation for gamma-ray bursts with a plateau phase in the afterglow
}

\author{
M. $\mathrm{Xu}^{1,2,3}$ and Y. F. Huang ${ }^{1,3}$ \\ 1 Department of Astronomy, Nanjing University, 210093 Nanjing, PR China \\ e-mail: hyf@nju.edu.cn \\ 2 Department of Physics, Yunnan University, 650091 Kunming, PR China \\ 3 Key Laboratory of Modern Astronomy and Astrophysics (Nanjing University), Ministry of Education, PR China
}

Received 22 July 2011 / Accepted 25 November 2011

\begin{abstract}
Aims. Gamma ray bursts (GRBs) offer strong advantages because of their huge burst energies, luminosities, and high redshifts in probing the Universe. A few interesting luminosity correlations of GRBs have been used to test cosmology models. Especially, for a subsample of long GRBs with known redshifts and a plateau phase in the afterglow, a correlation between the end time of the plateau phase (in the GRB rest frame) and the corresponding X-ray luminosity has been found. Here, we add the isotropic $\gamma$-ray energy release as a third parameter to get a tighter three-parameter correlation.

Methods. We reanalyzed the subsample and found that a significantly tighter correlation exists when we add the isotropic $\gamma$-ray energy release into the consideration. We used the Markov-chain Monte Carlo techniques to get the best-fit coefficients.

Results. A new three-parameter correlation is found for the GRBs with an obvious plateau phase in the afterglow. The best fit correlation is found to be $L_{\mathrm{X}} \propto T_{\mathrm{a}}^{-0.87} E_{\gamma, \text { iso }}^{0.88}$. Additionally, both long and intermediate duration GRBs are consistent with the same three-parameter correlation equation.

Conclusions. We argue that the new three-parameter correlation is consistent with the hypothesis that the subsample of GRBs with a plateau phase in the afterglow is associated with the birth of rapidly rotating magnetars and that the plateau is due to the continuous energy-injection from the magnetar. It is suggested that the newly born millisecond magnetars associated with GRBs might provide a good standard candle in the Universe.
\end{abstract}

Key words. ISM: jets and outflows - gamma-ray burst: general

\section{Introduction}

Gamma-ray busts (GRBs) are one of the most powerful and energetic explosive events in the Universe. The observations of GRBs up to redshifts higher than eight (Salvaterra et al. 2009; Cucchiara et al. 2011) cause GRBs to be among the farthest known astrophysical sources. Taking their considerable event rate into consideration, GRBs may be good candidates that can be used to probe our Universe. Several interesting correlations have been suggested for GRBs (Amati et al. 2002; Norris et al. 2000; Ghirlanda et al. 2004a; Liang \& Zhang 2005; Dainotti et al. 2010; Qi \& Lu 2010). Based on them, the cosmology parameters have been tentatively constrained (e.g., Fenimore \& Ramirez-Ruiz 2000; Schaefer 2003, 2007; Dai et al. 2004; Ghirlanda et al. 2004b, 2006; Amati et al. 2008; Wang \& Dai 2006; Dainotti et al. 2008; Wang et al. 2009, 2011).

To derive a meritorious constraint on the cosmology parameters, the most important thing is to find a credible standard candle relation for GRBs. Currently, no such a relation can be established when all GRBs are involved (Butler et al. 2009; Yu et al. 2009). The reason may be that different GRBs should be produced via various mechanisms. Interestingly, for a subsample of long GRBs with known redshifts and with a plateau phase in the afterglow, an anticorrelation has been reported to exist between the end time of the plateau phase $\left(T_{\mathrm{a}}\right.$, measured in the GRB rest frame) and the corresponding X-ray luminosity $\left(L_{X}\right)$ at that moment (Dainotti et al. 2010, hereafter D2010). In this paper, we denote the Dainotti et al. two-parameter correlation as the L-T correlation. The intrinsic scatter of this correlation is still too large to be directly applied as a redshift estimator (Dainotti et al. 2011). Additionally, normal long-duration GRBs and the intermediate duration GRBs do not obey the same correlation equation (D2010), and the intermediate class seems to be more scattered in the plot.

In this study, we have tried to add a third parameter, i.e. the isotropic $\gamma$-ray energy release $\left(E_{\gamma, \text { iso }}\right)$, into the correlation. We find that the new three-parameter correlation (designated as the L-T-E correlation) is much tighter than the previous L-T correlation. It is also obeyed by both the long GRBs and the intermediate-calss GRBs. The L-T-E correlation may hopefully give a better measure for our Universe. In Sect. 2, we describe our GRB sample and the method of data analysis. Our results are presented in Sect. 3. Section 4 contains our discussion and conclusions.

\section{Sample and data analysis}

According to Swift observations, many GRBs show a plateau phase in the early afterglow, prior to the normal power-law decay phase (Zhang et al. 2006; Nousek et al. 2006). In this study, we mainly concentrate on the GRBs with such a characteristics. All our GRBs are taken from the Dainotti et al. sample (D2010). In D2010's data table, a total of 77 GRBs are initially included, with known redshift and with a plateau phase in the afterglow light curve. After removing the intermediate-class GRBs and 
some GRBs with relatively large errors, they finally limited their major statics to only 62 long GRBs. Here, we have reselected the events by taking the following three criteria into account in our studies: (1) the plateau should be obvious (GRBs 050318, 050603, 060124, 060418, 061007, 070518, and 071031 are removed by us, since their phateau phase is not clear enough.); (2) the data in the plateau phase should be rich enough to show the profile of the plateau and the end time of the plateau as well (GRBs 050820A, 060512, 060904, and 060124 are removed by us due to this constraint.); and (3) there should be no flares during the plateau phase, since flares may affect the shape of the plateau light curve and lead to errors in the quantities that we are interested in (GRBs 050904, 050908, 060223A, and 060526 are removed by us according to this condition.). As a result, our "golden sample" consisted of 55 events in total, i.e., 47 long GRBs and eight intermediate-class GRBs. (Intermediate-class GRB are characterized by a short initial burst followed by an extended low intensity emission phase; Norris et al. 2006). The redshifts of our sample range from 0.08 to 8.26.

For the end times of the plateau phase $\left(T_{\mathrm{a}}\right.$, in the GRB rest frame) and the X-ray afterglow luminosities at that moment $\left(L_{\mathrm{X}} \equiv L_{\mathrm{X}}\left(T_{\mathrm{a}}\right)\right)$, we use the values of D2010. In D2010, $T_{\mathrm{a}}$ is derived through a phenomenological fitting model (Willingale et al. 2007), and $L_{X}$ is derived from the equation,

$L_{\mathrm{X}}=\frac{4 \pi D_{\mathrm{L}}^{2}(z) F_{\mathrm{X}}}{(1+z)^{1-\beta_{\mathrm{a}}}}$

where $z$ is the redshift, $D_{\mathrm{L}}(z)$ the luminosity distance, $F_{\mathrm{X}}$ the observed flux by $S w i f t-X R T$ at the end time of the plateau phase, and $\beta_{\mathrm{a}}$ the spectral index of the X-ray afterglow (Evans et al. 2009).

The isotropic $\gamma$-ray energy release in the prompt emission phase is

$E_{\gamma, \text { iso }}=4 \pi D_{\mathrm{L}}^{2}(z) S_{\text {bolo }} /(1+z)$,

where $S_{\text {bolo }}$ is the bolometric fluence, which can be taken from Wang et al. (2011). In this, $S_{\text {bolo }}$ is calculated from the observed energy spectrum $\Phi(E)$ as (Schaefer 2007):

$S_{\text {bolo }}=S \times \frac{\int_{1 /(1+z)}^{10^{4} /(1+z)} E \Phi(E) \mathrm{d} E}{\int_{E_{\min }}^{E_{\max }} E \Phi(E) \mathrm{d} E}$,

where $S$ is the observed fluence in units of $\mathrm{erg} \mathrm{cm}^{-2}$ for each $\mathrm{GRB}$, and $\left(E_{\min }, E_{\max }\right)$ are the detector thresholds. The energy spectrum $\Phi(E)$ is assumed to be the Band function (Band et al. 1993),

$\Phi(E)=\left\{\begin{array}{lc}A E^{\alpha} \mathrm{e}^{-(2+\alpha) E / E_{\text {peak }}} & E \leq \frac{\alpha-\beta}{2+\alpha} E_{\text {peak }} \\ B E^{\beta} & \text { otherwise }\end{array}\right.$

where $E_{\text {peak }}$ is the peak energy of the spectrum, and $\alpha, \beta$ are the power-law indices for photon energies below or above the break energy, respectively. Finally, the complete data set of all our 55 GRBs are shown in Table 1, where the error bars are in the $1 \sigma$ range.

We investigate whether an intrinsic correlation exists between the three parameters of $L_{\mathrm{X}}, T_{\mathrm{a}}$, and $E_{\gamma, \text { iso }}$ as

$\log \left(\frac{L_{\mathrm{X}}}{10^{47} \mathrm{erg} \mathrm{s}^{-1}}\right)=a+b \log \left(\frac{T_{\mathrm{a}}}{10^{3} \mathrm{~s}}\right)+c \log \left(\frac{E_{\gamma, \text { iso }}}{10^{53} \mathrm{erg}}\right)$,

where $a, b$, and $c$ are constants to be determined from the fit to the observational data. In this equation, $a$ is the constant of the intercept, while $b$ and $c$ are actually the power-law indices of time and energy when we approximate $L_{X}$ as power-law functions of $T_{\mathrm{a}}$ and $E_{\gamma \text {,iso. }}$. Due to the complexity of GRB sampling, an intrinsic scattering parameter, $\sigma_{\text {int }}$, is introduced in our analysis, as is usually done by other researchers (Reichart 2001; Guidorzi et al. 2006; Amati et al. 2008). This extra variable that follows a normal distribution of $N\left(0, \sigma_{\text {int }}^{2}\right)$ is engaged to represent all the contribution to $L_{\mathrm{X}}$ from other unknown hidden variables.

To derive the best fit to the observational data with the above three-parameter correlation, we use the method presented in $\mathrm{D}^{\prime}$ Agostini (2005). Here, to simplify, we first define $x_{1}=$ $\log \left(\frac{T_{\mathrm{a}}}{10^{3} \mathrm{~s}}\right), x_{2}=\log \left(\frac{E_{\gamma, \text { iso }}}{10^{53} \mathrm{erg}}\right)$, and $y=\log \left(\frac{L_{\mathrm{X}}}{10^{47} \mathrm{erg} / \mathrm{s}}\right)$. The joint likelihood function for the coefficients of $a, b, c$, and $\sigma_{\text {int }}$ is (D’Agostini 2005)

$$
\begin{aligned}
\mathcal{L}\left(a, b, c, \sigma_{\text {int }}\right) \propto \prod_{i} & \frac{1}{\sqrt{\sigma_{\text {int }}^{2}+\sigma_{y_{i}}^{2}+b^{2} \sigma_{x_{1, i}}^{2}+c^{2} \sigma_{x_{2, i}}^{2}}} \\
& \times \exp \left[-\frac{\left(y_{i}-a-b x_{1, i}-c x_{2, i}\right)^{2}}{2\left(\sigma_{\text {int }}^{2}+\sigma_{y_{i}}^{2}+b^{2} \sigma_{x_{1, i}}^{2}+c^{2} \sigma_{x_{2, i}}^{2}\right)}\right]
\end{aligned}
$$

where $i$ is the corresponding serial number of GRBs in our sample.

To get the best-fit coefficients, the so-called Markov-chain Monte Carlo techniques are used in our calculations. For each Markov chain, we generate $10^{6}$ samples according to the likelihood function. Then we derive the the coefficients of $a, b, c$, and $\sigma_{\text {int }}$ according to the statistical results of the samples.

Our likelihood function can also be conveniently applied to the two-parameter L-T correlation case studied by D2010, by simply taking $c=0$. We have checked our method by comparing our result for the L-T correlation with that of D2010. The results are generally consistent, which proves the reliability of our codes.

\section{Results}

In our study, we assume a flat $\Lambda \mathrm{CDM}$ cosmology with $H=$ $69.7 \mathrm{~km} \mathrm{~s}^{-1} \mathrm{Mpc}^{-1}$ and $\Omega_{\mathrm{M}}=0.291$ (the same values as D2010). By using the method described in Sect. 2, we find that the best-fit correlation between $L_{\mathrm{X}}, T_{\mathrm{a}}$ and $E_{\gamma \text {,iso }}$ is

$$
\log \left(\frac{L_{X}}{10^{47} \mathrm{erg} / \mathrm{s}}\right)=1.17-0.87 \log \left(\frac{T_{\mathrm{a}}}{10^{3} \mathrm{~s}}\right)+0.88 \log \left(\frac{E_{\gamma, \mathrm{iso}}}{10^{33} \mathrm{erg}}\right) .
$$

Figure 1 shows the above correlation. It is clearly shown that this three-parameter correlation is tight for all the 55 GRBs.

Comparing Eqs. (5) and (7), we find that the best values for the constants of $a, b$, and $c$ in Eq. (5) are $a=1.17, b=-0.87$, and $c=0.88$, respectively. Figure 1 also clearly shows that there is still obvious scatter in the L-T-E correlation. To give a quantitative description of the scatter, we need to derive the $1 \sigma$ errors of these constants.

The probability distributions of these constants, as well as the intrinsic scattering parameter $\left(\sigma_{\text {int }}\right)$, are displayed in Fig. 2. From this figure, we find that the probability distributions of these coefficients can be well fitted by Gauss functions, so we can easily get the $1 \sigma$ error bars for these parameters. Actually, the best values and the $1 \sigma$ errors for the coefficients are $a=$ $1.17 \pm 0.09, b=-0.87 \pm 0.09, c=0.88 \pm 0.08$, and $\sigma_{\text {int }}=$ $0.43 \pm 0.05$, respectively.

We have also explored the three-parameter correlation for all the 77 GRB events listed in D2010, using the same analytical method as for our "golden sample" of 55 GRBs. The 


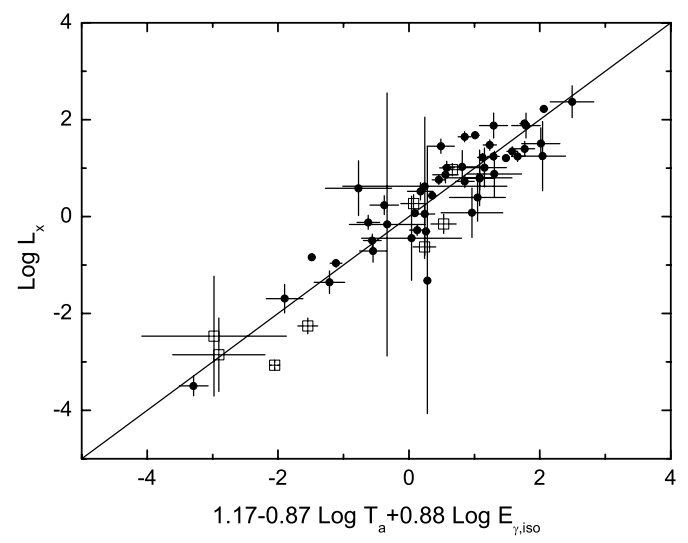

Fig. 1. The best-fit correlation between $L_{\mathrm{X}}, T_{\mathrm{a}}$, and $E_{\gamma, \text { iso }}$ for our "golden sample". $Y$-axis is the $\mathrm{X}$-ray luminosity at the end time of the plateau phase, i.e. $L_{X}$, in units of $10^{47} \mathrm{erg} / \mathrm{s}$. The $X$-axis is a combined quantity of $T_{\mathrm{a}}$ (in units of $10^{3} \mathrm{~s}$ ) and $E_{\gamma, \text { iso }}$ (in units of $10^{53} \mathrm{erg}$ ), i.e. $1.17-0.87 \log T_{\mathrm{a}}+0.88 \log E_{\gamma, \text { iso }}$. The filled points correspond to the observed data of 47 long GRBs and the hollow square points correspond to the eight intermediate-class GRBs. The solid line is plotted from Eq. (7), which is the best fit of the 55 observational data points.
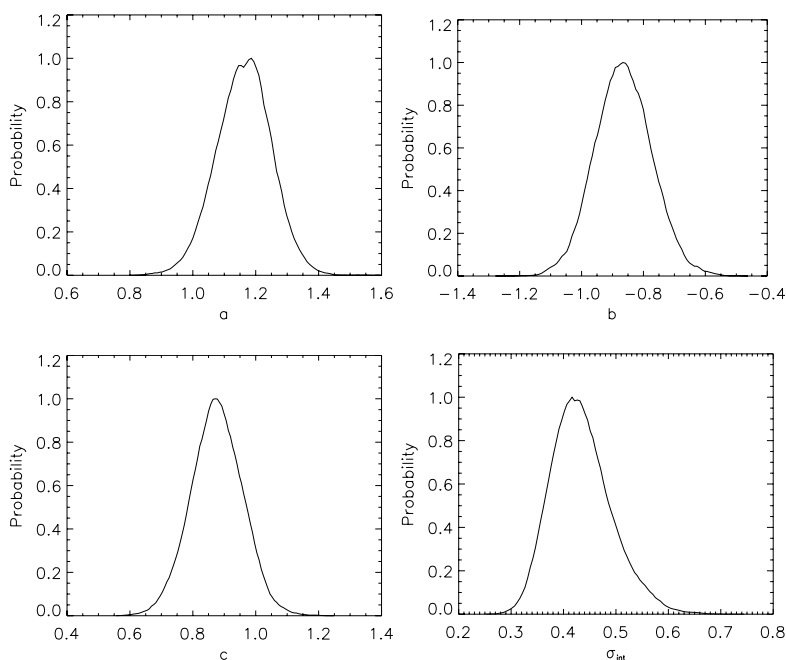

Fig. 2. The probability distributions of the constants of a (upper left panel), $b$ (upper right panel), $c$ (lower left panel) in Eq. (5), and the probability distribution of the intrinsic scattering parameter $\sigma_{\text {int }}$ (lower right panel). According to these panels, the best values and the $1 \sigma$ errors for the coefficients are $a=1.17 \pm 0.09, b=-0.87 \pm 0.09$, $c=0.88 \pm 0.08, \sigma_{\text {int }}=0.43 \pm 0.05$, respectively.

best-fit result is shown in Fig. 3. The best parameter values and the $1 \sigma$ errors for the coefficients are $a=0.81 \pm 0.07$, $b=-0.91 \pm 0.09, c=0.59 \pm 0.05$, and $\sigma_{\text {int }}=1.15 \pm 0.12$. Comparing with the result of the "golden sample", although there is still an obvious correlation among $L_{X}, T_{\mathrm{a}}$, and $E_{\gamma \text {,iso }}$ for all the 77 GRBs, the intrinsic scatter of the L-T-E correlation is much larger now. However, it is very important to note that we exclude the 22 samples because they most likely do not physically belong to the same group as the "golden sample" (for example, many of them do not have an obvious plateau phase), as judged from the three criteria in Sect. 2.

To directly compare with the L-T correlation suggested by D2010, we also fit the two-parameter correlation for our sample. The best-fit equation is

$$
\log \left(\frac{L_{\mathrm{X}}}{10^{47} \mathrm{erg} / \mathrm{s}}\right)=(0.78 \pm 0.14)-(1.16 \pm 0.16) \log \left(\frac{T_{\mathrm{a}}}{10^{3} \mathrm{~s}}\right) .
$$

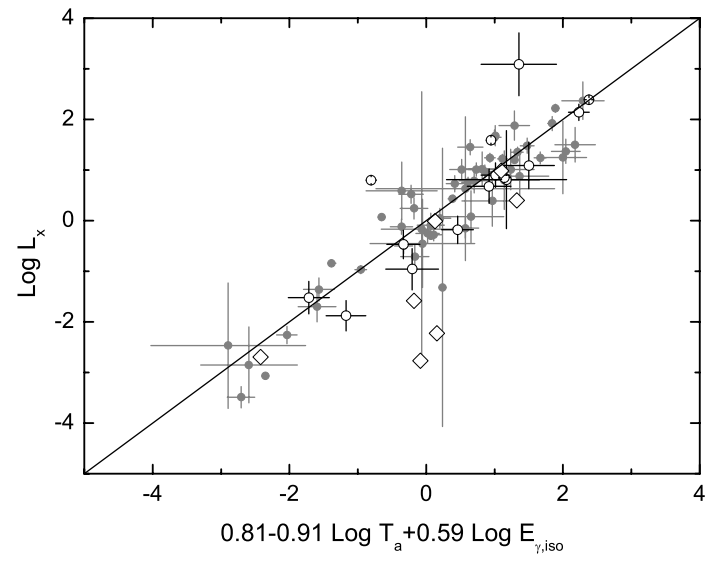

Fig. 3. The best-fit correlation between $L_{\mathrm{X}}, T_{\mathrm{a}}$ and $E_{\gamma, \text { iso }}$ for all the 77 GRBs of D2010. The units of all physical quantity are the same as Fig. 2. The $X$-axis is a combined quantity of $0.81-0.91 \log T_{\mathrm{a}}+$ $0.59 \log E_{\gamma, \text { iso }}$. The filled points correspond to the observed data of 55 "golden" GRBs with error bars. The hollow diamonds correspond to 7 GRBs with too large error bars to be plotted in the figure, and the hollow circles correspond to other 15 discarded events. The solid line is the best fit for all the 77 data points.

This equation is consistent with the L-T correlation derived in D2010. Comparing Eq. (8) with Eq. (7), and from Fig. 2, we find that the error bars of the constants in Eq. (8) (i.e. the L-T correlation) are generally significantly larger than those of Eq. (7) (i.e. the L-T-E correlation). Also, in the two-parameter fitting of Eq. (8), the intrinsic scatter is $0.85 \pm 0.10$, which is also markedly larger than in the three-parameter correlation case $(0.43 \pm 0.05)$. From the comparison, we see that the L-T-E correlation is really significantly tighter than the L-T correlation.

For our GRB sample, we also find that the correlation coefficient of our L-T-E statistics is $r=0.92$, and the chance probability is $P=1.05 \times 10^{-20}$. In contrast, the correlation coefficient of the L-T statistics of the same sample is $r=-0.73$ and the corresponding chance probability $P=5.55 \times 10^{-8}$. This also shows that the L-T-E correlation is much tighter than the L-T correlation.

\section{Discussion and conclusions}

In this paper, a new three-parameter correlation is found for the GRBs with an obvious plateau phase in the afterglow. This L-T-E correlation is tighter than the L-T correlation reported in D2010. It has been shown that the intrinsic scattering of our L-T-E correlation is significantly smaller than that of the L-T correlation, and the correlation coefficient is correspondingly larger. However, we note that the intrinsic scatter of the L-T-E correlation is still larger than that of some correlations derived from prompt GRB emission (Guidorzi et al. 2006; Amati et al. 2008). In the future, more samples and more delicate selections might help to improve the result.

The plateau phase (or the shallow decay segment) is an interesting characteristic of many GRB afterglows (Zhang et al. 2006; Nousek et al. 2006). This phenomenon can be explained as continuous energy injection from the central engine after the prompt burst (Rees \& Mészáros 1998; Dai \& Lu 1998; Zhang \& Mészáros 2001; Dai 2004; Kobayashi \& Zhang 2007; Yu \& Dai 2007; Xu et al. 2009; Yu et al. 2010; Dall'Osso et al. 2011) or by the two-component models (Corsi \& Mészáros 2009), or by structured jets (Eichler \& Granot 2006; Granot et al. 2006; Panaitescu 2007; Yamazaki 2009; Xu \& Huang 2010), or even 
Table 1. 55 GRBs of our sample.

\begin{tabular}{|c|c|c|c|c|c|}
\hline GRB & $z^{A}$ & $\log \left[L_{\mathrm{X}} /(\mathrm{erg} / \mathrm{s})\right]^{A}$ & $\log \left[T_{\mathrm{a}} /(\mathrm{s})\right]^{A}$ & $\log \left[E_{\gamma, \text { iso }} /(\mathrm{erg})\right]^{B}$ & Type $^{C}$ \\
\hline 050315 & 1.95 & $47.05 \pm 0.19$ & $3.92 \pm 0.17$ & $52.85 \pm 0.012$ & Long \\
\hline 050319 & 3.24 & $47.52 \pm 0.18$ & $4.04 \pm 0.17$ & $52.90 \pm 0.057$ & Long \\
\hline 050401 & 2.9 & $48.45 \pm 0.15$ & $3.28 \pm 0.14$ & $52.50 \pm 0.098$ & Long \\
\hline 050416A & 0.65 & $46.29 \pm 0.23$ & $2.97 \pm 0.21$ & $51.02 \pm 0.027$ & Long \\
\hline 050505 & 4.27 & $48.03 \pm 0.34$ & $3.67 \pm 0.33$ & $53.26 \pm 0.019$ & Long \\
\hline 050724 & 0.26 & $44.53 \pm 1.24$ & $4.92 \pm 1.22$ & $50.17 \pm 0.055$ & $\mathrm{IC}$ \\
\hline 050730 & 3.97 & $48.68 \pm 0.07$ & $3.44 \pm 0.04$ & $53.26 \pm 0.017$ & Long \\
\hline 050801 & 1.38 & $47.86 \pm 0.17$ & $2.17 \pm 0.16$ & $51.49 \pm 0.066$ & Long \\
\hline 050802 & 1.71 & $47.43 \pm 0.06$ & $3.52 \pm 0.06$ & $52.59 \pm 0.021$ & Long \\
\hline 050803 & 0.42 & $46.55 \pm 0.87$ & $2.74 \pm 0.81$ & $51.46 \pm 0.069$ & Long \\
\hline 050814 & 5.3 & $47.88 \pm 0.47$ & $3.13 \pm 0.45$ & $53.29 \pm 0.029$ & Long \\
\hline 050824 & 0.83 & $45.30 \pm 0.29$ & $4.65 \pm 0.27$ & $51.13 \pm 0.052$ & Long \\
\hline 050922C & 2.2 & $48.92 \pm 0.07$ & $2.08 \pm 0.07$ & $52.77 \pm 0.009$ & Long \\
\hline 051016B & 0.94 & $47.59 \pm 0.57$ & $3.22 \pm 0.55$ & $51.01 \pm 0.034$ & Long \\
\hline 051109A & 2.35 & $48.01 \pm 0.13$ & $3.4 \pm 0.11$ & $52.72 \pm 0.018$ & Long \\
\hline 051109B & 0.08 & $43.51 \pm 0.21$ & $3.64 \pm 0.19$ & $48.55 \pm 0.064$ & Long \\
\hline 051221A & 0.55 & $44.74 \pm 0.16$ & $4.51 \pm 0.16$ & $51.40 \pm 0.014$ & IC \\
\hline 060108 & 2.03 & $46.50 \pm 0.13$ & $3.92 \pm 0.13$ & $51.94 \pm 0.027$ & Long \\
\hline 060115 & 3.53 & $47.80 \pm 0.57$ & $3.09 \pm 0.55$ & $52.99 \pm 0.023$ & Long \\
\hline 060116 & 6.6 & $49.37 \pm 0.33$ & $1.8 \pm 0.3$ & $53.33 \pm 0.082$ & Long \\
\hline 060202 & 0.78 & $45.64 \pm 0.23$ & $4.74 \pm 0.23$ & $52.00 \pm 0.040$ & Long \\
\hline 060206 & 4.05 & $48.65 \pm 0.10$ & $3.15 \pm 0.1$ & $52.79 \pm 0.013$ & Long \\
\hline 060502A & 1.51 & $47.27 \pm 0.19$ & $3.85 \pm 0.21$ & $52.59 \pm 0.012$ & $\mathrm{IC}$ \\
\hline 060510B & 4.9 & $47.39 \pm 0.49$ & $3.78 \pm 0.48$ & $53.64 \pm 0.011$ & Long \\
\hline 060522 & 5.11 & $48.51 \pm 0.33$ & $2.07 \pm 0.31$ & $53.05 \pm 0.026$ & Long \\
\hline 060604 & 2.68 & $47.24 \pm 0.19$ & $3.98 \pm 0.18$ & $52.21 \pm 0.069$ & Long \\
\hline 060605 & 3.8 & $47.76 \pm 0.09$ & $3.48 \pm 0.08$ & $52.66 \pm 0.034$ & Long \\
\hline 060607A & 3.08 & $45.68 \pm 2.75$ & $4.14 \pm 0.02$ & $53.12 \pm 0.012$ & Long \\
\hline 060614 & 0.13 & $43.93 \pm 0.05$ & $5.01 \pm 0.05$ & $51.32 \pm 0.006$ & IC \\
\hline 060707 & 3.43 & $48.01 \pm 0.40$ & $2.94 \pm 0.36$ & $52.93 \pm 0.025$ & Long \\
\hline 060714 & 2.71 & $48.22 \pm 0.08$ & $3.11 \pm 0.07$ & $53.06 \pm 0.016$ & Long \\
\hline 060729 & 0.54 & $46.17 \pm 0.04$ & $4.73 \pm 0.04$ & $51.69 \pm 0.021$ & Long \\
\hline 060814 & 0.84 & $46.69 \pm 0.06$ & $4.01 \pm 0.06$ & $52.97 \pm 0.004$ & Long \\
\hline 060906 & 3.69 & $47.73 \pm 0.13$ & $3.62 \pm 0.12$ & $53.26 \pm 0.042$ & Long \\
\hline 060908 & 2.43 & $48.24 \pm 0.11$ & $2.46 \pm 0.09$ & $53.03 \pm 0.010$ & Long \\
\hline 060912A & 0.94 & $46.37 \pm 0.23$ & $2.97 \pm 0.18$ & $51.91 \pm 0.020$ & $\mathrm{IC}$ \\
\hline 061121 & 1.31 & $48.35 \pm 0.10$ & $3 \pm 0.09$ & $53.47 \pm 0.004$ & Long \\
\hline 070110 & 2.35 & $48.25 \pm 0.72$ & $1.89 \pm 0.37$ & $52.90 \pm 0.033$ & Long \\
\hline 070208 & 1.17 & $46.88 \pm 0.15$ & $3.63 \pm 0.14$ & $51.58 \pm 0.060$ & Long \\
\hline 070306 & 1.49 & $47.07 \pm 0.05$ & $4.42 \pm 0.04$ & $53.18 \pm 0.008$ & Long \\
\hline 070506 & 2.31 & $47.63 \pm 1.42$ & $2.87 \pm 1.42$ & $51.82 \pm 0.029$ & Long \\
\hline 070508 & 0.82 & $48.20 \pm 0.02$ & $2.75 \pm 0.02$ & $53.11 \pm 0.004$ & Long \\
\hline 070529 & 2.5 & $48.40 \pm 0.15$ & $2.34 \pm 0.15$ & $53.04 \pm 0.025$ & Long \\
\hline 070714B & 0.92 & $46.85 \pm 0.20$ & $3.03 \pm 0.19$ & $52.30 \pm 0.033$ & $\mathrm{IC}$ \\
\hline 070721B & 3.63 & $47.08 \pm 0.51$ & $3.58 \pm 0.51$ & $53.34 \pm 0.035$ & Long \\
\hline 070802 & 2.45 & $46.84 \pm 2.72$ & $3.68 \pm 0.62$ & $51.96 \pm 0.047$ & Long \\
\hline 070809 & 0.22 & $44.15 \pm 0.76$ & $4.09 \pm 0.75$ & $49.43 \pm 0.062$ & IC \\
\hline 070810A & 2.17 & $47.97 \pm 0.13$ & $2.83 \pm 0.12$ & $52.26 \pm 0.023$ & IC \\
\hline 071020 & 2.15 & $49.22 \pm 0.05$ & $1.84 \pm 0.05$ & $52.87 \pm 0.016$ & Long \\
\hline 080310 & 2.42 & $46.72 \pm 0.11$ & $4.08 \pm 0.11$ & $52.88 \pm 0.023$ & Long \\
\hline 080430 & 0.77 & $46.03 \pm 0.08$ & $4.29 \pm 0.08$ & $51.68 \pm 0.022$ & Long \\
\hline 080603B & 2.69 & $48.88 \pm 0.26$ & $2.92 \pm 0.24$ & $53.07 \pm 0.011$ & Long \\
\hline 080810 & 3.35 & $48.24 \pm 0.08$ & $3.28 \pm 0.07$ & $53.42 \pm 0.031$ & Long \\
\hline 081008 & 1.97 & $47.79 \pm 0.24$ & $2.95 \pm 0.22$ & $52.85 \pm 0.047$ & Long \\
\hline 090423 & 8.26 & $48.48 \pm 0.11$ & $2.95 \pm 0.1$ & $53.03 \pm 0.018$ & Long \\
\hline
\end{tabular}

Notes. All the error bars are in the $1 \sigma$ range. ${ }^{(A)}$ Taken from D2010. ${ }^{(B)}$ Calculated from Eq. $(2) .{ }^{(C)}$ Long means long GRB and IC is intermediateclass GRB.

as due to dust scattering (Shao \& Dai 2007; Shao et al. 2008). According to our L-T-E correlation (Eq. (7)), the X-ray luminosity at the end time of the plateau can be expressed as a function of the end time and the isotropic $\gamma$-ray energy release as

$L_{\mathrm{X}} \propto T_{\mathrm{a}}^{-0.87 \pm 0.09} E_{\gamma, \text { iso }}^{0.88 \pm 0.08}$
We believe that this relation can give useful constraints on the underlying physics.

For the energy injection model, a natural mechanism is the dipole radiation from the spinning down of a magnetar at the center of the fireball. Note that the injected energy may not be Poynting flux, but can be electron-positron pairs (Dai 2004). 
These pairs interact with the fireball material, leading to the formation of a relativistic wind bubble. When the energy injection dominates the dynamical evolution of the external shock, the afterglow intensity should naturally be proportional to the energy injection power, so $L_{X}$ is actually a measure of the energy injection rate. According to Eq. (9), $L_{X}$ is roughly inversely proportional to the timescale of the energy injection, $T_{\mathrm{a}}$. It hints that the energy reservoir should be roughly a constant. This is consistent with the energy injection model, which usually assumes that the central engine is a rapidly rotating millisecond magnetar. In different GRBs, the surface magnetic field intensities of the central magnetars may be quite different, leading to various energy injection luminosities and energy injection timescales. But the total energy available for energy injection is relatively constant (about rotational energy of the magnetar). It is mainly constrained by the limiting angular velocity of the magnetar, which again is determined by the equation of state of neutron stars. Additionally, according to Dai (2004), to produce an obvious plateau in the afterglow lightcurve, the total injected energy must be comparable to the original fireball energy (which may be comparable to $E_{\gamma, \text { iso }}$ ). This requirement is again roughly consistent with the item of $E_{\gamma, \text { iso }}^{0.88 \pm 0.08}$ in Eq. (9). Based on the above analyses, we argued that the L-T-E correlation strongly supports the energy injection model of magnetars. It also indicates that the newly born millisecond magnetars associated with GRBs provide a good standard candle in our Universe. Thus the L-T-E correlation may potentially be used to test the cosmological models.

Our sample contains 47 long GRBs and eight intermediateclass GRBs. From Fig. 1, we see that both of these two classes are consistent with the same L-T-E correlation. Howerer, they do behave very differently in frame work of the two-parameter L-T correlation. This is another important advantage of our threeparameter correlation. It indicates that magnetars may also form in intermediate-class GRBs, and their limiting spinning is simply similar to those magnetars born in long GRBs. A natural problem will be raised as to whether short GRBs with plateau phase in the afterglow also obey the same correlation. Unfortunately, the number of short GRBs meeting the requirement is currently too small.

It is worth noting that many interesting physical principles could be involved in newly born magnetars (Dall'Osso et al. 2009), which include the emission of gravitational waves, the cooling process, the evolution of the magnetic axis, etc. Some of the physics may affect the the energy injection process of the newly born magnetar delicately. We believe that further studies of the new three-parameter correlation may give useful constraints on the physics of newly born magnetars.

Acknowledgements. We thank the anonymous referee for many useful suggestions and comments. We also would like to thank Z. G. Dai, S. Qi, and F. Y. Wang for helpful discussion. This work was supported by the National Natural Science Foundation of China (Grant No. 11033002) and the National Basic Research Program of China (973 Program, Grant No. 2009CB824800).

\section{References}

Amati, L., Frontera, F., Tavani, M., et al. 2002, ApJ, 390, 81 Amati, L., Guidorzi, C., Frontera, F., et al. 2008, MNRAS, 391, 577

Band, D., Matteson, J., Ford, L., et al. 1993, ApJ, 413, 281

Butler, N. R., Kocevski, D., \& Bloom, J. S. 2009, ApJ, 694, 76

Corsi, A., \& Mészáros, P. 2009, ApJ, 702, 1171

Cucchiara, A., Levan, A. J., Fox, D. B., et al. 2011, ApJ, 736, 7

D'Agostini, G. 2005 [arXiv: astroph/0511182]

Dai, Z. G. 2004, ApJ, 606, 1000

Dai, Z. G., \& Lu, T. 1998, A\&A, 333, L87

Dai, Z. G., Liang, E. W., \& Xu, D. 2004, ApJ, 612, L101

Dainotti, M. G., Cardone, V. F., \& Capozziello, S. 2008, MNRAS, 391, L79

Dainotti, M. G., Willingale, R., Capozziello, S., Fabrizio Cardone, V., \& Ostrowski, M. 2010, ApJ, 722, L215

Dainotti, M. G., Cardone, V. F., Capozziello, S., Ostrowski, M., \& Willingale, R. 2011, ApJ, 730, 135

Dall' Osso, S., Shore, S. N., \& Stella, L. 2009, MNRAS, 398, 1869

Dall'Osso, S., Stratta, G., Guetta, D., et al. 2011, A\&A, 526, A121

Eichler, D., \& Granot, J. 2006, ApJ, 641, L5

Evans, P., Beardmore, A. P., Page, K. L., et al. 2009, MNRAS, 397, 1177

Fenimore, E. E., \& Ramirez-Ruiz, E. 2000 [arXiv: astro-ph/0004176]

Ghirlanda, G., Ghisellini, G., \& Lazzati, D. 2004a, ApJ, 616, 331

Ghirlanda, G., Ghisellini, G., Lazzati, D., \& Firmani, C. 2004b, ApJ, 613, L13

Ghirlanda, G., Ghisellini, G., \& Firmani, C. 2006, New J. Phys., 8, 123

Granot, J., Königl, A., \& Piran, T. 2006, MNRAS, 370, 1946

Guidorzi, C., Frontera, F., Montanari, E., et al. 2006, MNRAS, 371, 843

Kobayashi, S., \& Zhang, B. 2007, ApJ, 655, 973

Liang, E. W., \& Zhang, B. 2005, ApJ, 633, 611

Norris, J. P., \& Bonnell, J. T. 2006, A\&A, 643, 266

Norris, J. P., Marani, G. F., \& Bonnell, J. T. 2000, ApJ, 534, 248

Nousek, J. A., Kouveliotou, C., Grupe, D., et al. 2006, ApJ, 642, 389

Panaitescu, A. 2007, MNRAS, 379, 331

Qi, S., \& Lu, T. 2010, ApJ, 717, 1274

Rees, M. J., \& Mészáros, P. 1998, ApJ, 496, L1

Reichart, D. E. 2001, ApJ, 553, 235

Salvaterra, R., Della Valle, M., Campana, S., et al. 2009, Nature, 461, 1258

Schaefer, B. E. 2003, ApJ, 583, L67

Schaefer, B. E. 2007, ApJ, 660, 16

Shao, L., \& Dai, Z. G. 2007, ApJ, 660, 1319

Shao, L., Dai, Z. G., \& Mirabal, N. 2008, ApJ, 675, 507

Wang, F. Y., \& Dai, Z. G. 2006, MNRAS, 368, 371

Wang, F. Y., Dai, Z. G., \& Qi, S. 2009, A\&A, 507, 53

Wang, F. Y., Qi, S., \& Dai, Z. G. 2011, MNRAS, 415, 3423

Willingale, R. W., O'Brien, P. T., Osborne, J. P., et al. 2007, ApJ, 662, 1093

Xu, M., \& Huang, Y. F. 2010, A\&A, 523, 5

Xu, M., Huang, Y. F., \& Lu, T. 2009, RAA, 9, 1317

Yamazaki, R. 2009, ApJ, 690, L118

Yu, Y. W., \& Dai, Z. G. 2007, A\&A, 470, 119

Yu, B., Qi, S., \& Lu, T. 2009, ApJ, 705, 15

Yu, Y. W., Cheng, K. S., \& Cao, X. F. 2010, ApJ, 715, 477

Zhang, B., \& Mészáros, P. 2001, ApJ, 552, L35

Zhang, B., Fan, Y. Z., \& Dyks, J., et al. 2006, ApJ, 642, 354 